\title{
Francophonies d'Amérique
}

\section{Publications et thèses soutenues (2015)}

\section{Émilie LaFlèche}

Numéro 40-41, automne 2015, printemps 2016

URI : https://id.erudit.org/iderudit/1043716ar

DOI : https://doi.org/10.7202/1043716ar

Aller au sommaire du numéro

Éditeur(s)

Les Presses de l'Université d'Ottawa

Centre de recherche en civilisation canadienne-française

ISSN

1183-2487 (imprimé)

1710-1158 (numérique)

Découvrir la revue

Citer ce document

LaFlèche, É. (2015). Publications et thèses soutenues (2015). Francophonies

d'Amérique, (40-41), 289-301. https://doi.org/10.7202/1043716ar d'utilisation que vous pouvez consulter en ligne.

https://apropos.erudit.org/fr/usagers/politique-dutilisation/ 


\section{Publications et thèses soutenues (2015)}

\section{Émilie LaFlèche}

Université de Saint-Boniface

Les titres précédés d'un astérisque font l'objet d'une recension dans ce numéro.

\section{LIVRES}

Aвbal, Odon. Un rêve oublié entre Guyane et Brésil : la République de Counani, Matoury, Ibis Rouge Éditions, 2015, 112 p., coll. " Espace outre-mer ».

Alamkan, Myriam. Vous irez porter le fer et la flamme, Matoury, Ibis Rouge Éditions, 2015, 272 p., coll. "Espace outre-mer ».

Arcangeli, Myriam. Sherds of History: Domestic Life in Colonial Guadeloupe, Gainesville, University Press of Florida, 2015, 226 p.

Arsenault, Georges. Mémoires de l'honorable Aubin-Edmond Arsenault : ancien premier ministre et juge de la Cour suprême de l'Île-du-Prince-Édouard, TracadieSheila, Éditions La Grande Marée, 2015, 224 p.

Ayangma, Stanislas. L'aménagement territorial et ses effets sur le patrimoine des communautés autochtones de la Guyane française, Matoury, Ibis Rouge Éditions, 2015, 478 p., coll. " Espace outre-mer ».

Barzasi, Stéphanie. Haïti, l'histoire en héritage : le tremblement de terre du 12 janvier 2010 dans les récits de presse français, Paris, L'Harmattan, 2015, 264 p.

Basque, Philippe. Histoire de vies à Saint-Isidore, Lévis, Éditions de la Francophonie, 2015, 320 p., coll. " Mémoire vivante".

Benedicty-Kokken, Alessandra. Spirit Possession in French, Haitian, and Vodou Thought: An Intellectual History, Lanham, Lexington Books, 2015, 419 p. 
Benoist, Jean. Chronique d'un lieu de pensée : Fonds Saint-Jacques, Matoury, Ibis Rouge Éditions, 2015, 208 p., coll. « Espace outre-mer ».

Benoît, Catherine. Au cour des ténèbres de la friendly island : migrations, culture et sida à Saint-Martin, Québec, Les Presses de l'Université Laval, 2015, 230 p., coll. "Société, cultures et santé ».

Bergeaud, Émeric. Stella: A Novel of the Haitian Revolution, traduit et édité par Lesley S. Curtis et Christen Mucher, New York, New York University Press, 2015, 224 p.

Bernabé, Jean. Approche cognitive du créole martiniquais : Ranboulzay 1/Révolution 1, Paris, L'Harmattan, 2015, 242 p.

Bertrand, Luc. Trois histoires de bravoure : le Canada français et la Croix de Victoria, Québec, Les Presses de l'Université Laval, 2015, 218 p.

BILÉ, Serge. Esclave et bourreau : l'histoire incroyable de Mathieu Léveillé, esclave de Martinique devenu bourreau en Nouvelle-France, Québec, Éditions du Septentrion, 2015, $168 \mathrm{p}$.

Blamont, Jacques. Voilà la nouvelle université de Guyane-Numérique, Culture, Parc amazonien, Matoury, Ibis Rouge Édition, 2015, 284 p., coll. "Espace outre-mer ».

Bock, Michel, et François Charbonneau (dir.). Le siècle du Règlement 17 : regards sur une crise scolaire et nationale, Sudbury, Éditions Prise de parole, 2015, 472 p., coll. «Agora ".

Bonilla, Yarimar. Non-Sovereign Futures: French Caribbean Politics in the Wake of Disenchantment, Chicago, University of Chicago Press, 2015, 232 p.

Bosphore, Roland. Militants et militantismes communistes à la Martinique 19201971, Matoury, Ibis Rouge Éditions, 2015, 604 p., coll. « Espace outre-mer ».

Brunet, Guy. Mariage et métissage dans les sociétés coloniales: Amériques, Afrique et îles de l'océan Indien (XVI'-XX' siècles) = Marriage and Misgeneration in Colonial Societies: Americas, Africa and Islands of the Indian Ocean, $X V I^{\text {th }}-X X^{\text {th }}$ Centuries, Berne, Peter Lang, 2015, 358 p., coll. "Population, famille et société ».

CAIllot, Bernard. Lafayette, de l'Auvergne à l'Amérique (1757-1784), Paris, L'Harmattan, 2015, 262 p., coll. « Historiques ».

Calmont, André. Haïti entre permanences et ruptures, Matoury, Ibis Rouge Éditions, 2015, 604 p., coll. " Espace outre-mer». 
Cardinal, Linda, et Éric Forgues (dir.). Gouvernance communautaire et innovations au sein de la francophonie néobrunswickoise et ontarienne, Québec, Les Presses de l'Université Laval, 2015, 294 p., coll. «Langues officielles et sociétés ».

Delraciné, Vilmond Joegodson, et Paul Jackson. Rocks in the Water, Rocks in the Sun: A Memoir from the Heart of Haiti, Athabasca, Athabasca University Press, 2015, 340 p., coll. « Our Lives: Diary, Memoir, and Letters ».

Dessens, Nathalie. Creole City: A Chronicle of Early American New Orleans, Gainesville, University of Florida Press, 2015, 288 p.

Dugoujon, Casimir. Lettres sur l'esclavage et l'abolition dans les colonies françaises, 1840-1850, présentation de Nelly Schmidt, Paris, L'Harmattan, 2015, 256 p., coll. "Autrement Mêmes ».

Duguay, Louise. Pauline Boutal: An Artist's Destiny, 1894-1992, traduit vers l'anglais par S. E. Stewart, Winnipeg, University of Manitoba Press, 2015, 272 p.

Dumontet, Danielle. Les lieux d'oubli de la Francophonie, Hildesheim, Georg Olms Verlag, 2015, 228 p.

Durizot Jno-Baptiste, Paulette. Bilinguisme créole-français en milieu scolaire guadeloupéen, Paris, L'Harmattan, 2015, 206 p.

Duvivier, Max U. Trois études sur l'occupation américaine d'Haïti (1915-1934), Québec, Mémoire d'encrier, 2015, 112 p.

Dziembowski, Edmond. Les Pitt : l'Angleterre face à la France, 1708-1806, Québec, Éditions du Septentrion, 2015, 600 p.

Eybalin Casséus, Clara Rachel. Transnationalisme associatif haïtien et jamaïcain : géographie du développement local et politiques diasporiques, Saarbrücken, Éditions universitaires européennes, 2015, 292 p.

Fallon, Gérald, et André Élias Mazawi. Au-delà d'une école à deux vitesses : pour une école fondamentale juste et équitable en Haïti, Paris, L'Harmattan, 2015, 158 p., coll. " Documentation haïtienne ".

Fleurimond, Wiener Kerns. Haïti, l'état de la Nation, Matoury, Ibis Rouge Éditions, 2015, 428 p. coll. « Espace outre-mer ».

FLORY, Céline. De l'esclavage à la liberté forcée : histoire des travailleurs africains engagés dans la Caraïbe française au XIX $X^{e}$ siècle, Paris, Karthala, 2015, 456 p., coll. « Hommes et sociétés». 
FoncK, Bertrand, et Laurent Veyssière (dir.). La chute de la Nouvelle-France : de l'affaire Jumonville au traité de Paris, Québec, Éditions du Septentrion, 2015, $586 \mathrm{p}$.

Fournier, Marcel. Les Français émigrés au Canada pendant la Révolution française et le Consulat, 1789-1804, Québec, Éditions du Septentrion, 2015, 282 p.

Frith, Nicola, et Kate Hodgson. At the Limits of Memory: Legacies of Slavery in the Francophone World, Don Mills, Oxford University Press, 2015, 256 p., coll. «Francophone Postcolonial Studies ».

Gauthériot, Murielle. L'entreprise Beauport à la Guadeloupe : un exemple d'aménagement territorial et de transformation sociale, Paris, L'Harmattan, 2015, 212 p.

Gordon, Lewis R. What Fanon Said: A Philosophical Introduction to His Life and Thought, New York City, Fordham University Press, 2015, 216 p.

Guyot, Philippe. Paroles de poilus guyanais : propos, lettres et carnets de la Grande Guerre, 1914-1918, Matoury, Ibis Rouge Éditions, 2015, 104 p., coll. « Histoire et patrimoine des outre-mer ".

* Hallion, Sandrine, Bertrand Nayet et Charles Leblanc (dir.). Voix, portraits de douze auteurs, Winnipeg, Éditions du Blé, 2015, 344 p.

HaYday, Matthew. So They Want Us to Learn French: Promoting and Opposing Bilingualism in English-Speaking Canada, Vancouver, University of British Columbia Press, 2015, 304 p.

* Нотте, Lucie (dir.). René Dionne et Gabrielle Poulin : auvres et vies croisées, Ottawa, Éditions David, 2015, 266 p.

JoHn, Berthela. Le tambour dans la culture des jeunes Créoles guyanais, Matoury, Ibis Rouge Éditions, 2015, 144 p., coll. « Espace outre-mer ».

Kellman, Jordan (dir.). Lafayette in Transnational Context: Identity, Travel, and Nationalism in the Revolutionary Atlantic World, Lafayette, University of Louisiana at Lafayette Press, 2015, 156 p.

KLein, Alexandre, et Séverine PARAYRe (dir.). Histoire de la santé XVIII ${ }^{e}-X X^{e}$ siècles : nouvelles recherches francophones, Québec, Les Presses de l'Université Laval, 2015, $242 \mathrm{p}$.

LABRIDY, Lorène. Flux et langues en milieu urbain créole : étude de sociolinguistique à Fort-de France, Paris, L'Harmattan, 2015, 214 p., coll. « Espaces discursifs ». 
LAFERrière, Dany. Dany Laferrière à l'Académie française : discours de réception, Montréal, Éditions du Boréal, 2015, 84 p.

LAmbert, Steve. "Aller de l'avant" : chroniques d'un libre penseur acadien, t. II, Lévis, Éditions de la Francophonie, 2015, 166 p.

LANDry, Michelle. L'Acadie politique : histoire sociopolitique de l'Acadie du Nouveau-Brunswick, Québec, Les Presses de l'Université Laval, 2015, 170 p., coll. « Langues officielles et sociétés ».

Landry, Michelle, Martin PÂQuet et Anne Gilbert (dir.). Mémoires et mobilisations, Québec, Les Presses de l'Université Laval, 2015, 318 p., coll. « Culture française d'Amérique ».

Lonergan, David. Théâtre l'Escaouette, 1977-2012, Sudbury, Éditions Prise de parole, 2015, $403 \mathrm{p}$.

Mam Lam Fouck, Serge. La société guyanaise à l'épreuve des migrations : 19652015, Matoury, Ibis Rouge Éditions, 2015, 154 p., coll. « Espace outre-mer ».

Manne, Laura. Les Antilles et la Guyane en 365 dates, Lamentin (Martinique), Caraïbéditions, 2015, $144 \mathrm{p}$.

McIntosh, Malachi. Emigration and Caribbean Literature, New York, Palgrave Macmillan, 2015, 256 p., coll. « New Caribbean Studies ».

Mignot, Dominique Aimé, et Jean-Gabriel Montauban. Esclavage et servitude aux Antilles : l'héritage antique et médiéval -XVII $-X X^{e}$, Paris, L'Harmattan, 2015, $372 \mathrm{p}$.

Moomou, Jean. Sociétés marronnes des Amériques, Matoury, Ibis Rouge Éditions, 2015, 784 p., coll. «Espace outre-mer ».

Nolette, Nicole. Jouer la traduction : théâtre et hétérolinguisme au Canada francophone, Ottawa, Les Presses de l'Université d'Ottawa, 2015, 300 p., coll. « Regards sur la traduction ».

Pamphile, Leon D. Contrary Destinies: A Century of America's Occupation, Deoccupation, Reoccupation of Haiti, Gainesville, University Press of Florida, 2015, $222 \mathrm{p}$.

Rogers, Dominique. Voix d'esclaves : Antilles, Guyane et Louisiane françaises, XVIII ${ }^{e}$ XIX ${ }^{e}$ siècle, Paris, Karthala, 2015, 192 p., coll. " Esclavages ". 
Sanschagrin, David. Les juges contre le Parlement? La conscience politique de l'Ouest et la contre-révolution des droits au Canada, Québec, Les Presses de l'Université Laval, 2015, 188 p.

Schnakenbourg, Christian. Histoire de l'industrie sucrière en Guadeloupe aux $X I X^{e}$ et XX $X^{e}$ siècles : les "Vingt glorieuses» de la sucrerie guadeloupéenne (1946-1965), Paris, L'Harmattan, 2015, 176 p.

Ternat, François. Partager le monde : rivalités impériales franco-britanniques (1748-1756), Paris, Presses de l'université Paris-Sorbonne, 2015, 584 p.

Thonhauser, Brigitte. Les traces de la civilisation française en Louisiane et la naissance du mythe cajun, Saarbrücken, Éditions universitaires européennes, 2015, $132 \mathrm{p}$.

VIAU, Robert. Acadie multipiste : romans acadiens, t. 1, Moncton, Éditions PerceNeige, 2015, 228 p., coll. " Essais et documents ».

* Warren, Jean-Philippe. Honoré Beaugrand: la plume et l'épée (1848-1906), Montréal, Éditions du Boréal, 2015, 532 p.

Zonzon, Jacqueline. Les mutations de la société coloniale guyanaise, de l'abolition à la départementalisation 1848-1946, Matoury, Ibis Rouge Éditions, 2015, 128 p., coll. « Le jeune historien guyanais ».

Zouari, Ilyes. Petit dictionnaire du Monde francophone : tout ce quil faut savoir sur les 45 pays et territoires francophones, Paris, L'Harmattan, 2015, 456 p.

\section{THÈSES}

Akpossan, Johanne. La consonne / R/ comme indice de la variation lectale : cas du français en contact avec le créole guadeloupéen, thèse de doctorat, Paris, Université Sorbonne Nouvelle, 2015.

Arsenec, Nicole. Étude contrastive des créoles de la Jamaïque et de la Martinique, thèse de doctorat, Marseille, Université Aix-Marseille, 2015.

Augustin-Billy, Andia. Bodies in Transgression: Exploring Same-Sex Relations in Contemporary Francophone Caribbean Literature, thèse de doctorat, St. Louis, Washington University in St. Louis, 2015.

Bassong-DesRochers, Lova. La négritude dans la littérature afro-caribéenne contemporaine : mort ou transformation?, thèse de maitrise, Victoria, University of Victoria, 2015. 
Blanc, Judite. Evénement sismique de 2010 en Haïti et psychopathologies : facteurs de risques pre, peri et post-traumatiques, thèse de doctorat, Paris, Université de Paris 13 Nord, 2015.

Boniface, Philippe. La mobilité professionnelle des Martiniquais dans la Caraïbe : analyse de la situation, enjeux, propositions, thèse de doctorat, Pointe-à-Pitre, Université des Antilles et de la Guyane, 2015.

Bonner, Christopher T. The Alignment of Writing: Cold War Geopolitics and Literary Form in Francophone Caribbean Literature, thèse de doctorat, New York City, New York University, 2015.

Boucher, Michelle. Le "care " et l'éthique du " care" chez les directions d'écoles de langue française pluralistes de la région Centre de l'Ontario, thèse de doctorat, Ottawa, Université d'Ottawa, 2015.

Boudreau, Eugénie. Invisibilisation et revalorisation : transformations du sens et des pratiques dans la culture économique en contexte acadien du nord-est $d u$ Nouveau-Brunswick, thèse de maitrise, Ottawa, Université d'Ottawa, 2015.

Boutouchent, Fadila. Apprentissage et maintien du français langue seconde ou langue additionnelle : une étude auprès de jeunes canadiens anglophones, thèse de doctorat, Moncton, Université de Moncton, 2015.

Brant, Daniel. Geographies of Suffering: The Literature of Catastrophe in the Francophone Caribbean, thèse de doctorat, University of Illinois at UrbanaChampaign, 2015.

Burke-Saulnier, Andrea J. Could You Say That in "bon français", Please? How Students Experience Oral Corrective Feedback in Minority French Language Classrooms, thèse de doctorat, Minneapolis, Capella University, 2015.

Buteau, Emmanuel. Encountering God in Haiti: An Invitation to Postcolonial Practical Theology, thèse de doctorat, Miami Gardens, St. Thomas University, 2015.

Camp, Albert. L'essentiel ou Lagniappe: The Ideology of French Revitalization in Louisiana, thèse de doctorat, Bâton Rouge, Louisiana State University, 2015.

CampbelL-Hewitt, Laurie. Mère et fils, homme et femme dans la littérature antillaise, thèse de doctorat, Lafayette, University of Louisiana at Lafayette, 2015.

CANDAU, Olivier-Serge. Les alternances de langues en accompagnementpersonnalisé: des situations éducatives aux processus interactionnels : l'exemple de Saint-martin, thèse de doctorat, Pointe-à-Pitre, Université des Antilles et de la Guyane, 2015. 
CAPRICORne, Sandra. Impact des contes et des récits endogènes sur la performance des compétences d'écriture d'élèves de sixième en diffculté en espace créolophone martiniquais : approche narrato-discursive et psychoculturelle, thèse de doctorat, Pointe-à-Pitre, Université des Antilles et de la Guyane, 2015.

Chacon, Roman F. Neocolonial Crossroads: An Intercultural History of Haiti and the United States during the Interwar Era, thèse de maitrise, Flagstaff, Northern Arizona University, 2015.

Champlain-Corriveau, Simon-Pierre. Entre démocratisation et tradition : la modernisation de l'enseignement en français à l'école bilingue franco-ontarienne (1927-1960), thèse de maîtrise, Ottawa, Université d'Ottawa, 2015.

Chopin, Pierre. Prototypage de mosaïques de systèmes de culture répondant à des enjeux de développement durable des territoires : application à la Guadeloupe, thèse de doctorat, Pointe-à-Pitre, Université des Antilles et de la Guyane, 2015.

Cidalise-Montaise, Marie-Dominique. Communication dans le système éducatif martiniquais et exclusion sociale, thèse de doctorat, Pointe-à-Pitre, Université des Antilles et de la Guyane, 2015.

Coco, Roger. Le régime juridique de prévention et de réparation des risques naturels majeurs face aux exigences de justice sociale : le cas des Antilles françaises, thèse de doctorat, Pointe-à-Pitre, Université des Antilles et de la Guyane, 2015.

Concy, Huguette. La transmission du patrimoine médicinal créole : problématique, pertinence et évaluation d'un savoir traditionnel, thèse de doctorat, Pointe-à-Pitre, Université des Antilles et de la Guyane, 2015.

CôtÉ, Kristy A. Resilience and Quality of Life for Francophone Patients Living With Prostate Cancer: An Analysis of the Situation in Northern Ontario, Canada, thèse de doctorat, Sudbury, Université Laurentienne, 2015.

COURRENT, Lauwencia. La problématisation migratoire dans l'ultrapériphérie européenne : étude d'une fenêtre d'opportunité à travers les cas de la Guadeloupe et des Canaries, thèse de doctorat, Pointe-à-Pitre, Université des Antilles et de la Guyane, 2015.

Des Becquets, Magdalena. La réception critique du théatre acadien et francoontarien entre identité et modernité, thèse de maîtrise, Ottawa, Université d'Ottawa, 2015.

Dickinson, Christine. Aspects of Performativity in New Orleans Voodoo, thèse de maîtrise, Washington, George Washington University, 2015. 
Dubors, Janique F. "Just Do It"! Self-Determination for Complex Minorities, thèse de doctorat, Toronto, University of Toronto, 2015.

Durban-Albrecht, Erin L. Postcolonial Homophobia: United States Imperialism in Haiti and the Transnational Circulation of Antigay Sexual Politics, thèse de doctorat, Tuscon, University of Arizona, 2015.

Eulis, Elizabeth N. The Many Ties of the Petites Nations: Relationships, Power, and Diplomacy in the Lower Mississippi Valley, 1685-1788, thèse de doctorat, Chapel Hill, The University of North Carolina at Chapel Hill, 2015.

Gamess, Eline. Propositions pour une mixité réussie entre les hommes et les femmes dans les sphères de décision et de direction : étude comparative entre la Martinique et le Québec, thèse de doctorat, Pointe-à-Pitre, Université des Antilles et de la Guyane, 2015.

GARNIER, Claire. Soin des corps, soin des âmes : genre et pouvoir dans les hôpitaux de France et de Nouvelle-France aux XVII ${ }^{e}$ et XVIII ${ }^{e}$ siècles, thèse de doctorat, Montréal, Université de Montréal, 2015.

Godin, Gilberte. Liens entre certaines caractéristiques linguistiques, culturelles et psychologiques du personnel enseignant en contexte francophone minoritaire au Canada et ses pratiques pédagogiques, thèse de doctorat, Moncton, Université de Moncton, 2015.

Gordien, Ary. Nationalisme, race et ethnicité en Guadeloupe : constructions identitaires ambivalentes en situation de dépendance, thèse de doctorat, Paris, Université Paris Descartes, 2015.

Gotin, Jacques. Education thérapeutique du patient asthmatique bilingue françaiscréole en Guadeloupe : quels outils, quel impact?, thèse de doctorat, Pointe-à-Pitre, Université des Antilles et de la Guyane, 2015.

Gour, Rochelle. Ontario Middle-School Core French Teachers' Perceptions of and Experiences with the 2013 Ontario French as a Second Language Curriculum, thèse de maîtrise, Toronto, University of Toronto, 2015.

Gow, Jamella Nefetari. "The Country in Our Minds": Diasporic Longing, Ethnic Solidarity and Political Consciousness within the Haitian Transnational Community, thèse de maitrise, Santa Barbara, University of California Santa Barbara, 2015.

Haskins, Meredith B. French Immersion in Louisiana: Instructor Perceptions and Practices, thèse de maîtrise, Tuscaloosa, University of Alabama, 2015. 
Heestand, Pamela. The Spaces of Memory in the Early Works of Jose Maria Arguedas and Édouard Glissant, thèse de doctorat, Davis, University of California Davis, 2015.

Huston, Kristin Nicole. "Something at Least Human": Transatlantic (Re)Presentations of Creole Women in Nineteenth-Century Literature and Culture, thèse de doctorat, Kansas City, University of Missouri-Kansas City, 2015.

Jolicoeve, Manon. Limpact de cercles de lecture au sein d'équipes de hockey de garçons de neuf et dix ans du Nouveau-Brunswick francophone, thèse de doctorat, Moncton, Université de Moncton, 2015.

Jones Wilson, Jennifer C. H. The Impact of French Opera in Nineteenth-Century New York: The New Orleans French Opera Company, 1827-1845, thèse de doctorat, New York City, The City University of New York, 2015.

Jung, Marjorie. La figure de l'homme nouveau dans l'œuvre de Frantz Fanon : la décolonisation de lêtre au prisme de la social-thérapie textuelle, thèse de doctorat, Paris, Université Paris-Sorbonne, 2015.

JuRaWAN, Kimberley. "Toxic Island " et "L'Empreinte à Crusoe " : l'individuation de lidentité franco-antillaise?, thèse de maittrise, Boca Raton, Florida Atlantic University, 2015.

Kamali Kageruka, Eddy. La diversité ethnoculturelle dans les manuels de sciences du secondaire de l'Ontario, thèse de maîtrise, Ottawa, Université d'Ottawa, 2015.

KaMBLÉ, Sangita. Le choix scolaire des parents immigrants et l'engagement au français de leurs enfants, thèse de doctorat, Ottawa, Université d'Ottawa, 2015.

KARIRE, Blandine. La compétence interculturelle : une nécessité incontournable en milieu scolaire franco-ontarien, thèse de maîtrise, Sudbury, Université Laurentienne, 2015.

KNIGHT, Catherine. Les difficultés et les stratégies d'insertion en emploi des immigrants haïtiens dans la région d'Ottawa-Gatineau, thèse de maitrise, Ottawa, Université d'Ottawa, 2015.

Krenka, Klaudia. The Acquisition of Grammatical Gender by French as a Second Language Learners Enrolled in French Immersion, thèse de maittrise, Toronto, University of Toronto, 2015.

LABelle, Hélène. Le traitement lexicographique du français canadien dans Usito, dictionnaire québécois informatisé, thèse de maîtrise, Ottawa, Université d'Ottawa, 2015. 
LACELle, Suzanne. Les jeunes femmes de l'Ontario français et le féminisme, mémoire de maitrise, Sudbury, Université Laurentienne, 2015.

LaMothe, Mario Jacques. On Dedouble: Haiti, Contemporary Dance, and Performances of Vodou and Voodoo, thèse de doctorat, Ann Arbor, Northwestern University, 2015.

Lanoux, Gloria Martha. The Traiteur: Giving Voice to Traditional Cajun Healing in Louisiana, thèse de doctorat, Monroe, University of Louisiana at Monroe, 2015.

Lauro-Raoulx, Raphaël. Édouard Glissant : penseur du monde, poète de la terre, thèse de doctorat, Paris, Université Paris Ouest Nanterre, 2015.

Lepage, Andrée. The Contribution of Word Stress and Vowel Reduction to the Intelligibility of the Speech of Canadian French Second Language Learners of English, thèse de doctorat, Québec, Université Laval, 2015.

Marcelin, Angelot. Description et analyse des retombées du PCBF sur l'administration publique haïtienne, mémoire de maîtrise, Chicoutimi, Université du Québec à Chicoutimi, 2015.

Marius, Philippe-Richard. Privilege in Haiti: Travails in Color of the First Bourgeois Nation-State in the Americas, thèse de doctorat, New York, The City University of New York, 2015.

Misran, Jennifer. L'identité en question : espace(s) et perception de soi dans la littérature francophone africaine et caribéenne contemporaine, thèse de doctorat, Madison, University of Wisconsin - Madison, 2015.

Morphis, Elizabeth Aphrodite. Becoming Writers: Young Emergent Bilinguals' Multiple Worlds and Literacies in a French-English Curriculum, thèse de doctorat, New York, Columbia University, 2015.

Moussavou, Emeric. La quête de l'identité dans le roman francophone postcolonial: approche comparée des littératures africaine, insulaire, maghrébine et caribéenne, thèse de doctorat, Limoges, Université de Limoges, 2015.

Mueller, Katherine Elizabeth. Rethinking Pedagogical Options for Teaching Explicit Grammar in French Immersion, thèse de doctorat, Calgary, University of Calgary, 2015.

NADEAU, Josée. Adaptation et validation de l'ODEDYS pour les élèves francophones de $5^{e}$ année du Nouveau-Brunswick, thèse de doctorat, Moncton, Université de Moncton, 2015. 
NG, E-Ching. The Phonology of Contact: Creole Sound Change in Context, thèse de doctorat, New Haven, Yale University, 2015.

PAuzé, Elise. La qualité de l'alimentation et l'accès alimentaire des ménages vulnérables habitant dans une zone d'intervention d'agriculture de santé publique en Haïti, thèse de maîtrise, Ottawa, Université d'Ottawa, 2015.

Pennik, Patricia Jean. Exotic Folk: Old-Time Louisiana Music and the Politics of Culture, 1946-1973, thèse de doctorat, Boston, Boston University, 2015.

Peters, Jason. The Sentinelle Affair: A Study in Multilingual Language Practices, thèse de doctorat, Kingston, University of Rhode Island, 2015.

Pierre-Louis, Jessica. Les Libres de couleur face au préjugé : franchir la barrière à la Martinique aux XVII ${ }^{e}$-XVIII ${ }^{e}$ siècles, thèse de doctorat, Pointe-à-Pitre, Université des Antilles et de la Guyane, 2015.

Rabalais, Nathan Joseph. Contes rendus: Sources and Development of Lousiana's French and Creole Oral Tradition, thèse de doctorat, New Orleans, Tulane University, 2015.

Rehill, Anne Collier. Coureurs de bois, Backwoodsmen As Ecocritical Motif in Four Works of French Canadian Literature, thèse de doctorat, College Park, University of Maryland, 2015.

Remy, Avonelle Pauline. Infiltrating the Colonial City through the Imaginaries of Metissage: Saint-Louis (Senegal), Saint-Pierre (Martinique) and Jeremie (Haiti), thèse de doctorat, Iowa City, University of Iowa, 2015.

Riвo, John D. Decolonizing the Caribbean Borderlands: The Haitian Revolution in Contemporary Latinola Cultural Production, thèse de doctorat, Chapel Hill, University of North Carolina at Chapel Hill, 2015.

Richer, Geneviève. Entre loyauté partisane et engagement nationaliste : NapoléonAntoine Belcourt, le Parti libéral et la cause canadienne-française, 1860-1932, thèse de doctorat, Ottawa, Université d'Ottawa, 2015.

Royer, Zéphrine. Propositions pour un enseignement bilingue préélémentaire : enquêtes et expérimentations à la Martinique et à la Dominique, thèse de doctorat, Pointe-à-Pitre, Université des Antilles et de la Guyane, 2015.

Schmitт, Romain. Analyse de la validité prédictive d'une épreuve standardisée de langue française chez des étudiants locuteurs natifs francophones inscrits dans des programmes de formation en enseignement : le cas du TFLM, thèse de maîtrise, Québec, Université Laval, 2015. 
Scoтt, Lindsey. Imagining Women and Sexuality under Duvalier: $21^{\text {st-Century }}$ Representations of the Duvalier Regimes in Novels by Haitian Women, thèse de doctorat, Tallahassee, Florida State University, 2015.

SHADD, Deborah. On Language, Education and Identity: Minority Language Education within the Canadian Context, thèse de doctorat, Ottawa, University of Ottawa, 2015.

Sмiтн, Emily Carol Helen. Teachers' Perspectives on Plurilingualism in the French Immersion Program, thèse de maîtrise, Toronto, University of Toronto, 2015.

SohaIL, Juwairia. The Within- and Cross-Language Role of Syntactic Awareness in Reading Comprehension Among French Immersion Students, thèse de maitrise, Toronto, University of Toronto, 2015.

Tancons, Gauthier. "Moi mangrove" de "l'amor mundis" comme écosophie pour un enseignement actionnable et enseignable du patrimoine immatériel culturel en Guadeloupe : une épistémologie du vivre ensemble laïc en aire créolophone : l'esclavage, l'éducation, la franc-maçonnerie, thèse de doctorat, Perpignan, Université de Perpignan, 2015.

Vagalau, Eliana. Nomadic Memory in the Contemporary French Caribbean Novel, thèse de doctorat, Evanston, Northwestern University, 2015.

VIERGINAT ANDRÉ, Joslyne. Regards croisés sur la relation école-famille et la réussite scolaire d'élèves à l'école fondamentale en Haïti, thèse de doctorat, Montréal, Université de Montréal, 2015.

VItal, Louise Michelle. Tet Chage! An Investigation of the Haitian Education and Leadership Program in the Higher Education Landscape in Haiti, thèse de doctorat, Ann Arbor, Michigan State University, 2015.

Willard, Ashley. Engendering Islands: Representations of Difference in the Seventeenth-Century French Caribbean, thèse de doctorat, New York, The City University of New York, 2015.

Wood, Sarah Louise. Tensions of Development and Negotiations of Identity at the Periphery of France: Guyane Française since 1946, thèse de doctorat, Manchester, University of Manchester, 2015. 